\title{
Possible involvement of T helper type 2 responses to Toll-like receptor ligands in IgG4-related sclerosing disease.
}

\section{$\operatorname{AUTHOR}(\mathrm{S})$ :}

Akitake, Reiko; Watanabe, Tomohiro; Zaima, Chikage; Uza, Norimitsu; Ida, Hiroshi; Tada, Shinsuke; Nishida, Naoshi; Chiba, Tsutomu

\section{CITATION:}

Akitake, Reiko ... [et al]. Possible involvement of T helper type 2 responses to Toll-like receptor ligands in IgG4-related sclerosing disease.. Gut 2010, 59(4): 542-545

\section{ISSUE DATE:}

2010-04

URL:

http://hdl.handle.net/2433/120797

RIGHT:

(C) 2010 BMJ Publishing Group Ltd \& British Society of Gastroenterology 


\section{GUT \\ Possible involvement of T helper type 2 responses to Toll-like receptor ligands in IgG4-related sclerosing disease}

Reiko Akitake, Tomohiro Watanabe, Chikage Zaima, et al.

Gut 2010 59: 542-545

doi: 10.1136/gut.2009.200972

Updated information and services can be found at:

http://gut.bmj.com/content/59/4/542.full.html

These include:

References This article cites 15 articles, 4 of which can be accessed free at: http://gut.bmj.com/content/59/4/542.full.html\#ref-list-1

Email alerting Receive free email alerts when new articles cite this article. Sign up in the service box at the top right corner of the online article.

Notes

To order reprints of this article go to:

http://gut.bmj.com/cgi/reprintform

To subscribe to Gut go to:

http://gut.bmj.com/subscriptions 


\title{
Possible involvement of T helper type 2 responses to Toll-like receptor ligands in IgG4-related sclerosing disease
}

\author{
Reiko Akitake, Tomohiro Watanabe, Chikage Zaima, Norimitsu Uza, Hiroshi Ida, \\ Shinsuke Tada, Naoshi Nishida, Tsutomu Chiba
}

Department of Gastroenterology and Hepatology, Kyoto University Graduate School of Medicine, Kyoto, Japan

\section{Correspondence to}

Dr Tomohiro Watanabe, Department of Gastroenterology and Hepatology, Kyoto University Graduate School of Medicine, 54 Shogoin Kawahara-cho, Sakyo-ku, Kyoto 606-8507, Japan; tmhrwtnb@kuhp.kyoto-u.ac.jp

Revised 4 December 2009 Accepted 22 December 2009

\section{ABSTRACT}

We report a case of immunoglobulin G4 (lgG4)-related sclerosing disease involving the pancreas, liver and salivary glands. Massive infiltration of IgG4-expressing plasma cells was seen in the liver and submandibular lymph nodes. Interestingly, accumulation of IgG4expressing plasma cells was also seen in the colon and terminal ileum. Peripheral blood mononuclear cells (PBMCs) isolated from this patient exhibited enhanced production of lgG4 and interleukin-10 upon stimulation with Toll-like receptor (TLR) ligands as compared with those from a healthy control. In contrast, production of tumour necrosis factor $\alpha$ and interferon $\gamma$ by PBMCs from this patient was markedly reduced. Since colonic mucosa is always exposed to TLR ligands derived from commensal organisms, the results of immunological studies suggest that enhanced T helper type 2 responses to intestinal microflora may underlie the immunopathogenesis in this patient with lgG4-related sclerosing disease.

\section{INTRODUCTION}

Autoimmune pancreatitis (AIP) is an inflammatory disorder which is characterised by increased serum levels of immunoglobulin G4 (IgG4) or by an IgG4positive plasmacytic infiltrate into the inflamed tissue. ${ }^{1}$ Another important feature of AIP is a wide variety of extrapancreatic manifestations such as sialadenitis, cholangitis, retroperitoneal fibrosis and inflammatory pseudotumour of the liver and lung. ${ }^{2}$ Since these extrapancreatic and pancreatic lesions share common histopathological findings (ie, abundant infiltration by $\operatorname{IgG} 4^{+}$plasmacytes), Kamisawa et al proposed a new clinicopathological entity: 'IgG4-related sclerosing disease'. ${ }^{2}$ However, little is understood regarding the role played by this $\operatorname{IgG}$ subtype in the inflammatory process. In this regard, IgG4 itself does not seem to be responsible for the development of tissue damage since this $\operatorname{IgG}$ subtype does not cause cell-mediated lysis due to poor binding activity to complement. ${ }^{3}$ In addition, anti-inflammatory activity of IgG4 has been shown. ${ }^{4}$ Consistent with these biological functions of IgG4, clinical manifestations of immune complex disease such as arthritis and glomerulonephritis are rarely seen in patients with IgG4-related sclerosing disease. ${ }^{5}$ These facts suggest that abnormal immunological environments leading to enhanced IgG4 responses, rather than IgG4 antibody itself, underlie the pathogenesis of this disease.
Distribution of IgG4-expressing plasmacytes in the gastrointestinal tract of patients with AIP has been observed. ${ }^{67}$ However, it is unknown whether this distribution of $\mathrm{IgG}^{+}$cells is directly induced by immune reactions occurring in the gastrointestinal tract or is indirectly induced by systemic IgG4 responses. Given the fact that mucosa of the gastrointestinal tract is always exposed to antigens derived from intestinal microflora, it is tempting to speculate that immune responses against microbial antigens create abnormal environments leading to enhanced IgG4 responses in the gut. Indeed, we experienced a case of IgG4-related sclerosing disease in which accumulation of IgG4-expressing plasmacytes was visualised as colonic inflammatory pseudopolyps. $^{8}$ Here we report a case with IgG4related sclerosing disease whose ileal and colonic mucosa bore a marked infiltration of IgG4expressing plasma cells. Interestingly, peripheral blood mononuclear cells (PBMCs) isolated from this case show enhanced T helper type 2 (Th2) and IgG4 responses upon stimulation with Toll-like receptor (TLR) ligands. These results indicate possible involvement of excessive Th2 responses against intestinal microflora in some cases with IgG4-related sclerosing disease.

\section{CASE REPORT}

A 70-year-old asymptomatic man was admitted for further investigation of swelling of the pancreas and submandibular lymph nodes. He had a history of systemic lymphadenopathy of unknown aetiology at the age of 45 . Laboratory tests revealed elevation of serum levels of amylase (229 IU/1; normal range <129 IU/l) and IgG $(2144 \mathrm{mg} / \mathrm{dl}$; normal range $<1840 \mathrm{mg} / \mathrm{dl}$ ). Abdominal CT using contrast reagent showed focal swelling of the pancreatic head without an enhancement effect. Endoscopic retrograde cholangiopancreatography revealed irregular narrowing of the main pancreatic duct and the stricture of the lower bile duct. These radiographic findings were consistent with those of AIP. ${ }^{1}$ A hypoechoic tumour was detected in the lateral segment of the liver on abdominal ultrasonography. Since a marked elevation of serum IgG4 level was detected $(918 \mathrm{mg} / \mathrm{dl}$; normal range $<135 \mathrm{mg} / \mathrm{dl}$ ), this patient was strongly suspected to have IgG4-related screlosing disease involving the pancreas, bile duct and liver. Biopsy of the liver tumour revealed massive infiltration of plasmacytes expressing IgG and IgG4 around the bile duct (figure 1). More than $50 \%$ of IgG-expressing cells 
were positive for IgG4 staining, which suggests that this liver tumour was a pseudotumour due to IgG4-associated cholangitis. Similar histological findings were obtained in the immunohistochemical analyses using biopsy specimens from submandibular lymph nodes (figure 1). Based on these results, this patient was finally diagnosed as having IgG4-related sclerosing disease.

Colonoscopy was performed to exclude the involvement of the gastrointestinal tract before starting prednisolone treatment. Although no inflammatory mucosa was seen from the terminal ileum to the rectum on colonoscopic examination, biopsy specimens taken from the intact mucosa of the terminal ileum and colon revealed a marked infiltration of plasmacytes expressing IgG into the submucosa without destruction of crypt architecture or fibrosis (figure 1). Interestingly, $>50 \%$ of IgGexpressing cells were positive for IgG4 staining. Accumulation of IgG4-expressing plasma cells in the colonic mucosa led us to hypothesise that abnormal immunological responses to gut microbial antigens might underlie the development of enhanced
IgG4 responses. PBMCs from this case and healthy controls were stimulated with TLR ligands to see immune responses against antigens derived from intestinal microflora. ${ }^{9}$ Ethical permission for this study was granted by the review board of Kyoto University. As shown in figure 2, production of IgG4 as well as interleukin-10 (IL-10) was enhanced upon stimulation with TLR4 (lipopolysaccharide (LPS)) and TLR5 (flagellin) ligands. Production of IgG4 was also enhanced by stimulation of a TLR2 ligand (peptidoglycan (PGN)). In contrast, production of Th1 cytokines (interferon $\gamma$ (IFN $\gamma$ ) and tumour necrosis factor $\alpha(\mathrm{TNF} \alpha)$ ) in response to TLR ligands by the patient's PBMCs was impaired as compared with that by control PBMCs. No difference was seen in the production of IL-8 or IL-17 upon stimulation with TLR ligands or TNF $\alpha$. These data suggest that activation of TLRs generates both IgG4 and Th2 responses in PBMCs from this case since IFN $\gamma$ and IL-10 are prototypical Th1 and Th2 cytokines, respectively. ${ }^{9}$ We determined the type of immune cells producing these cytokines by cell depletion study
Figure 1 Immunohistochemical staining of immunoglobulin G4 (lgG4) and $\operatorname{lgG}$. Biopsy specimens obtained from the liver, submandibular lymph nodes, terminal ileum and colon were stained with anti-lgG4 or anti-IgG antibody for visualisation of plasma cells expressing $\lg \mathrm{G} 4$ or $\lg \mathrm{G}$.
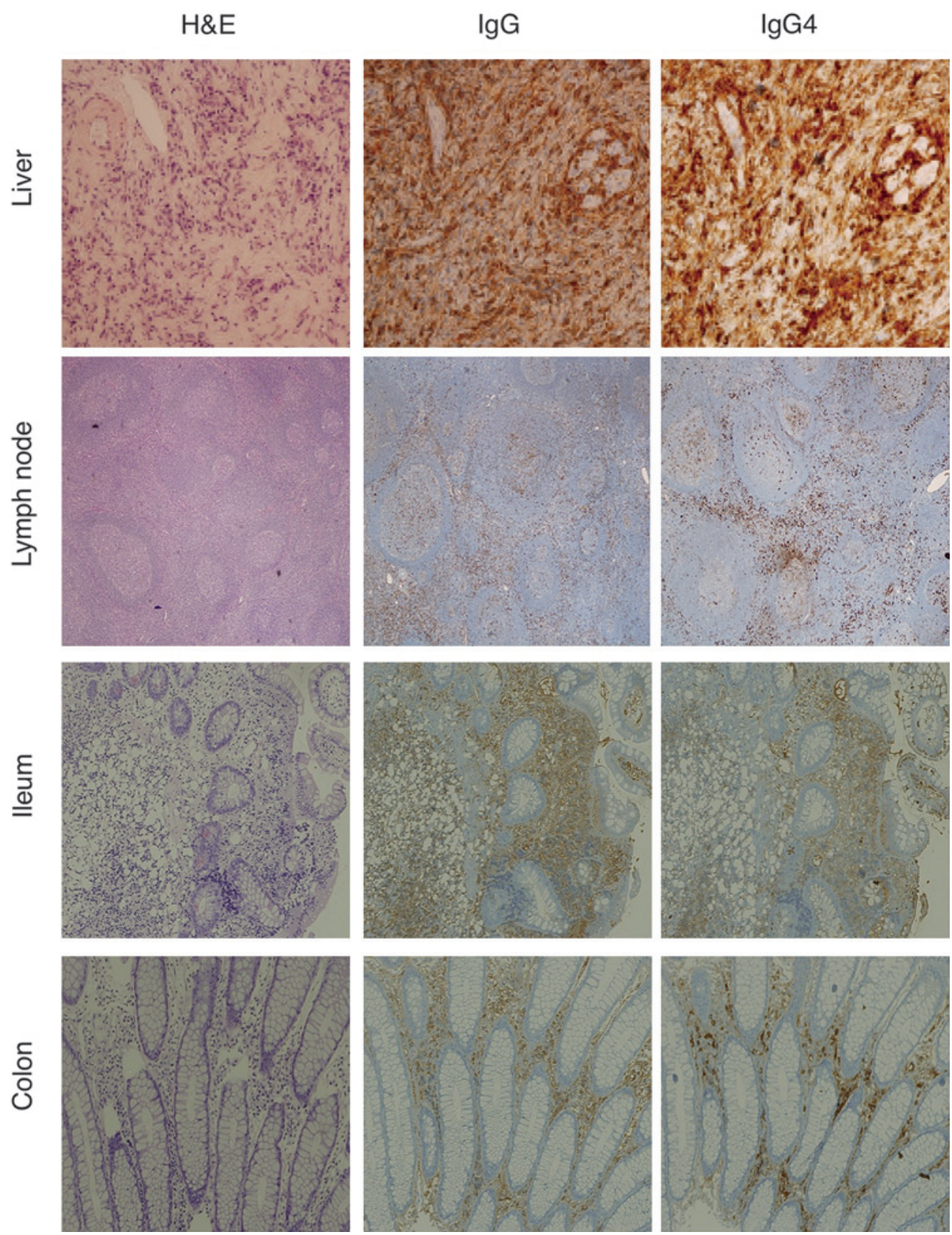
Figure 2 Enhanced T helper type 2 (Th2) responses to Toll-like receptor (TLR) ligands by peripheral blood mononuclear cells (PBMCs) isolated from the patient. PBMCs $\left(2 \times 10^{6} / \mathrm{ml}\right)$ isolated from the patient and healthy controls were stimulated with peptidoglycan (PGN, $10 \mu \mathrm{g} / \mathrm{ml}$ ), lipopolysaccharide (LPS, $1 \mu \mathrm{g} / \mathrm{ml}$ ), flagellin $(1 \mu \mathrm{g} / \mathrm{ml})$ or tumour necrosis factor (TNF, $20 \mathrm{ng} / \mathrm{ml})$. PBMCs were cultured for $48 \mathrm{~h}$ for interleukin-8 (IL-8) and TNF assay, and for 14 days for IgG4, interferon $\gamma$ (IFN $\gamma$ ), IL-10 and IL-17 assay. Results shown are means of triplicate wells.
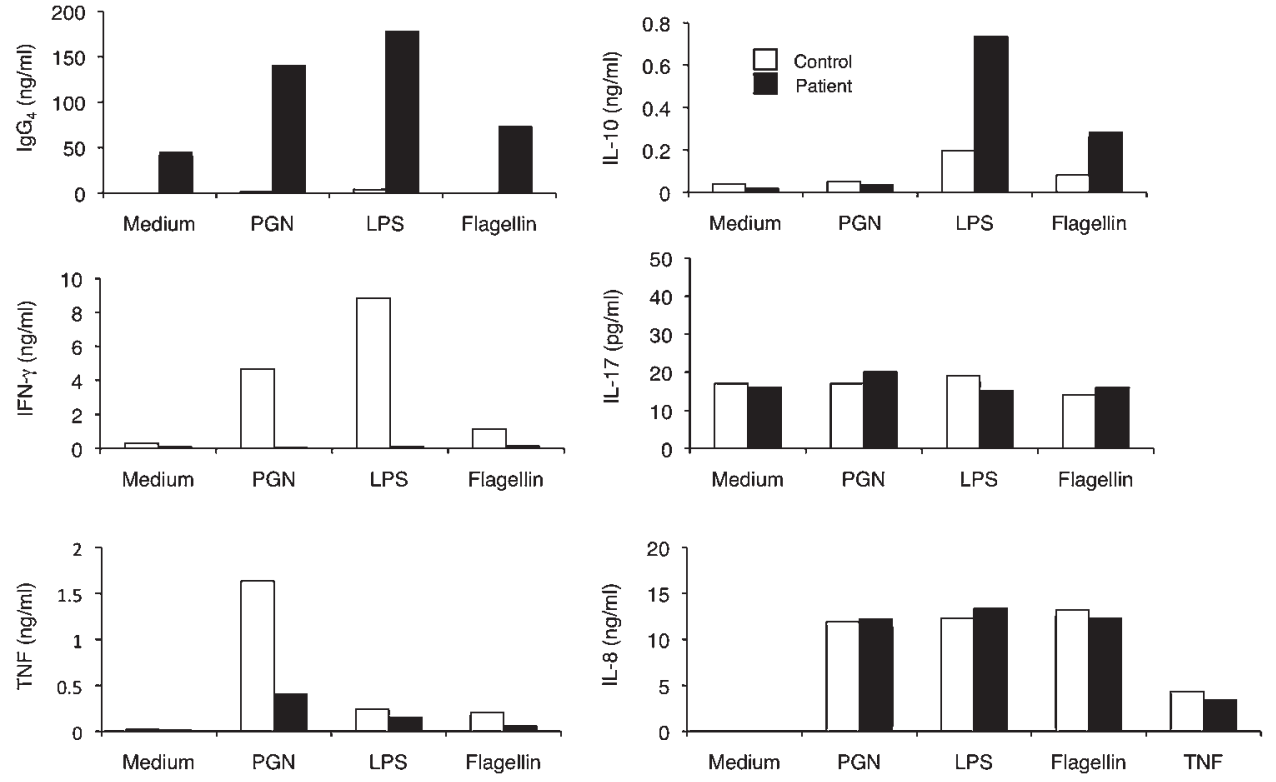

using control samples. We found that $\mathrm{CD}^{+} \mathrm{T}$ cells produced IFN $\gamma$ and IL-10 whereas CD14 ${ }^{+}$monocytes produced IL-10 and TNF $\alpha$ (data not shown).

\section{DISCUSSION}

An interesting observation in this case with IgG4-related sclerosing disease was a marked infiltration of IgG4-expressing plasmacytes into the colonic mucosa which appeared to be normal on endoscopic examination. It remains unclear whether we can regard this case as IgG4-related sclerosing disease involving the colonic mucosa since no pathological findings were present in colonic biopsy specimens other than marked infiltration of $\mathrm{IgG}^{+}$cells. Thus, unlike our previous case in which infiltration of IgG4-expressing plasmacytes was visualised as colonic polypoidal lesions, ${ }^{8}$ we have to be cautious in the interpretation of infiltration of IgG4-expressing plasma cells into endoscopically normal colonic mucosa in the setting of IgG4related sclerosing disease.

Immune responses leading to accumulation of IgG4expressing plasmacytes in the gastrointestinal tract are poorly understood. PBMCs isolated from this case exhibited enhanced production of IgG4 and Th2 cytokines upon stimulation with TLR ligands, suggesting that enhanced immune reactions against microbial antigens cause infiltration of lymphocytes as in the case of inflammatory bowel disease (IBD). ${ }^{10}$ In fact, this idea is supported by clinical evidence that a significant population of patients with AIP have a diagnosis of IBD. ${ }^{11}$ Importantly, IgG4 responses induced by TLR activation are associated with enhanced IL-10 production. In this regard, two different groups report involvement of regulatory $\mathrm{T}$ cells (Tregs) producing IL-10 in IgG4-related sclerosing disease. ${ }^{12} 13$ Thus, enhanced IL-10 production seen in this case may be partially mediated by circulating Tregs. Given the fact that IL-10 is an important cytokine for IgG4 class switching, ${ }^{14}$ we assume that excessive Th2 responses triggered by TLR ligands together with activation of Tregs create abnormal immunological environments leading to enhanced IgG4 responses. This idea partially explains immunological mechanisms of enhanced Th2 responses in patients with IgG4-related sclerosing disease. ${ }^{12}$

Although storiform fibrosis is a characteristic pathological finding of IgG4-related sclerosing disease, ${ }^{2}$ molecular mecha- nisms of fibrosis in this disorder are poorly understood. Th2 cytokines mediated by activation of TLRs on macrophages have been identified as positive regulators of tissue fibrosis in the liver and lung. ${ }^{15}$ Thus, enhanced Th2 responses to TLR ligands might be involved in the development of storiform fibrosis in IgG4related sclerosing disease. However, analysis of expression of both Th2 cytokines and TLRs using fibrotic tissue samples is necessary to address this issue.

What is the mechanism by which enhanced Th2 responses against intestinal microflora cause IgG4-related sclerosing disease without the development of colitis? In this regard, immune reactions causing tissue injury and those causing IgG4 responses should be considered separately as shown by the fact that IgG4 antibody itself has anti-inflammatory properties. ${ }^{4}$ Indeed, tissue destruction was not seen in the lower gastrointestinal tract of this case despite a marked infiltration of IgG4-expressing plasmacytes into the submucosa. Several mechanisms for preventing hyper-responsiveness to microbial antigens operate in the gut. For example, the intestine is the preferential site where naïve $\mathrm{T}$ cells differentiate into Tregs. ${ }^{10}$ Thus, one possible explanation is that pathogenic immune reactions causing tissue injury are suppressed in the gut by regulatory mechanisms, whereas such reactions cause tissue injury in other sterile organs such as the pancreas and bile duct due to the lack of regulatory mechanisms. Based on this, it is tempting to speculate that the gastrointestinal tract is an induction site for systemic IgG4 responses and functions as a reservoir for IgG4-expressing plasmacytes even if disease-associated pathogenic changes are absent. Alternatively, distribution of IgG4-expressing plasmacytes into the colonic mucosa may be an epiphenomenon associated with systemic IgG4 responses.

In conclusion, the results of immunological studies using PBMCs from this case suggest involvement of excessive Th2 responses to intestinal microflora in the development of IgG4related sclerosing disease. Confirmation of this idea awaits further studies using a large number of patients with IgG4related sclerosing disease.

Acknowledgements This work is supported in part by grants from Takeda Science Foundation, Pancreas Research Foundation of Japan, Uehara Memorial Foundation (to TW) and Health and Labour Sciences Research Grants for research on intractable diseases from Ministry of Health, Labour and Welfare of Japan (to TC). 


\section{Competing interests None.}

Ethics approval This study was conducted with the approval of the Kyoto University review board.

Patient consent Obtained.

Provenance and peer review Not commissioned; externally peer reviewed.

\section{REFERENCES}

1. Finkelberg DL, Sahani D, Deshpande V, et al. Autoimmune pancreatitis. N Engl J Med 2006;355:2670-6.

2. Kamisawa T, Okamoto A. Autoimmune pancreatitis: proposal of IgG4-related sclerosing disease. J Gastroenterol 2006;41:613-25.

3. Kawa S, Kitahara K, Hamano H, et al. A novel immunoglobulin-immunoglobulin interaction in autoimmunity. PLoS One 2008;3:e1637.

4. van der Neut Kolfschoten M, Schuurman J, Losen M, et al. Anti-inflammatory activity of human IgG4 antibodies by dynamic Fab arm exchange. Science 2007:317:1554-7.

5. Okazaki K, Chiba T. Autoimmune related pancreatitis. Gut 2002;51:1-4.

6. Kamisawa T, Egawa N, Nakajima $\mathrm{H}$, et al. Gastrointestinal findings in patients with autoimmune pancreatitis. Endoscopy 2005:37:1127-30.
7. Deheragoda MG, Church $\mathrm{NI}$, Rodriguez-Justo $\mathrm{M}$, et al. The use of immunoglobulin g4 immunostaining in diagnosing pancreatic and extrapancreatic involvement in autoimmune pancreatitis. Clin Gastroenterol Hepatol 2007:5:1229-34.

8. Ueno K, Watanabe T, Kawata $Y$, et al. IgG4-related autoimmune pancreatitis involving the colonic mucosa. Eur J Gastroenterol Hepatol 2008;20:1118-21.

9. Watanabe T, Asano N, Murray PJ, et al. Muramyl dipeptide activation of nucleotidebinding oligomerization domain 2 protects mice from experimental colitis. J Clin Invest 2008;118:545-59.

10. Strober W, Fuss I, Mannon P. The fundamental basis of inflammatory bowel disease. J Clin Invest 2007;117:514-21.

11. Ravi K, Chari ST, Vege SS, et al. Inflammatory bowel disease in the setting of autoimmune pancreatitis. Inflamm Bowel Dis 2009;15:1326-30.

12. Zen Y, Fujii T, Harada K, et al. Th2 and regulatory immune reactions are increased in immunoglobin G4-related sclerosing pancreatitis and cholangitis. Hepatology 2007:45:1538-46.

13. Miyoshi $\mathbf{H}$, Uchida K, Taniguchi $\mathrm{T}$, et al. Circulating naive and CD4+CD25high regulatory $T$ cells in patients with autoimmune pancreatitis. Pancreas 2008;36:133-40.

14. Jeannin $\mathbf{P}$, Lecoanet $\mathrm{S}$, Delneste $\mathrm{Y}$, et al. IgE versus IgG4 production can be differentially regulated by IL-10. J Immunol 1998:160:3555-61.

15. Wynn TA. Common and unique mechanisms regulate fibrosis in various fibroproliferative diseases. J Clin Invest 2007:117:524-9. 